\title{
Aproximación a la lógica tecnológica en la arquitectura contemporánea
}

\author{
An approach to technological logic in contemporary architecture
}

<RESUMEN>

Los arquitectos y académicos Alberto Montealegre B y José Solís 0 nos entregan una interesante mirada sobre la presencia de nuevas herramientas tecnológicas en el desarrollo del proceso de diseño arquitectónico y la necesidad de fortalecer instancias críticas y reflexivas dentro del ámbito universitario frente a un tema que parece dominar a la arquitectura contemporánea.

$<A B S T R A C T>$

The architects and academics Alberto Montealegre B and José Solis o. provide us with an interesting look at the presence of new technological tools in the process of architectural design, as well as the need to strengthen critical and reflexive bodies within the scope of the university concerning an issue that, at present, seems to dominate contemporary architecture.

<PALABRAS CLAVE >

TECNOLOGÍA / DISEÑO / ESTÉTICA / OBJETO / GLOBALIZACIÓN Y PERIFERIA

KKEY WORDS

TECHINOLOGY \& DESIGN \& AESTHETIS OBJECT । GLOBALISATION AND PERIPHERY

\section{Introducción}

La permanente consolidación de nuevas herramientas y medios tecnológicos capaces de influenciar y por momentos definir las metodologías de acción en el ámbito arquitectónico, permiten iniciar la reflexión sobre el verdadero alcance de estas aparentes nuevas prácticas y el papel que cumplen y ejercen sobre el proceso de diseño arquitectónico tal y como lo solíamos aprender y conocer.

La evidente fascinación que bien sabe acompañar la presencia de tales nuevas herramientas deberá ser cuestionada por un juicio crítico que nos permita dilucidar, o al menos acercarnos, a los códigos que definen la presencia arquitectónica en el mundo contemporáneo. En tal escenario Revista De Arquitectura ha invitado a participar de una conversación abierta y reflexiva a los arquitectos Alberto Montealegre B. y José Solís $0 .{ }^{1}$, ambos académicos comprometidos con el permanente intercambio de ideas sobre la contingencia de la arquitectura y su implicancia en el medio formativo universitario.

La primera interrogante que abre la discusión, es el genuino estado de correspondencia que puede llegar a existir entre métodos y herramientas basadas en parámetros tecnológicos propios del primer mundo y un país como Chile, donde la realidad cotidiana parece indicar una distancia no menor que salvar, frente a los modelos arquitectónicos desarrollados en un contexto de avanzada en términos tecnológicos. 
-Alberto Montealegre B.: Sin lugar a dudas hay un extremo de la producción arquitectónica, en tal escenario, que está realmente lejos de la realidad chilena, no sólo por una cuestión presupuestaria, sino por consideraciones técnicas y también culturales. Diferencia cultural, porque el tipo de clientes que posee cierta arquitectura del primer mundo son económicamente poderosos, están dispuestos a pagar por una arquitectura que interesa por el impacto que es capaz de producir, por la exploración formal, por la originalidad. Diferencia material porque son capaces de permitirse edificios muy difíciles de interpretar como elementos bidimensionales tradicionales de representación planimétrica, con espacios interiores de una gratuidad presupuestaria impresionante.

Cuando uno se plantea profesionalmente en ese contexto, las destrezas que uno tendría que tener son la capacidad de inventar cosas raras, cosas nuevas, de ponerse en situaciones inéditas, lo que es totalmente distinto a las destrezas en las que se sitúa un arquitecto latinoamericano, donde yo también creo que tienen sentido, por lo menos teóricamente, hablar de estos modelos tridimensionales que incorporan cada vez más información y que pretenden manejar sistemas de variables de manera compleja, porque en la situación del tercer o del segundo mundo, tenemos una cantidad de variables que no sólo son puramente funcionales y arquitectónicas, sino que son políticas, étnicas, etc. Entonces, podríamos tener la capacidad de integrar más cosas para satisfacer con nuestras obras, más necesidades que las presentes en el Primer Mundo, que se puede dar el lujo de tener un edificio que está más bien comprometido con una imagen corporativa lejana, que con el verdadero uso y buen desempeño funcional del espacio productivo.

—José Solís 0.: Yo quiero darle un giro a la reflexión. Me gustaría empezar con una cita muy conocida que trabaja Hegel en sus Lecciones de Estética, específicamente en la introducción y que se ha cifrado como el concepto de «muerte del arte», noción bastante conocida y que se desprende del siguiente modo: "Bajo todos estos aspectos, el arte por lo que se refiere a su destino supremo es y permanece para nosotros en un mundo pasado. Con ello también se ha perdido para nosotros la auténtica verdad y vitalidad. Si antes afirmaba su necesidad en la realidad y ocupaba el lugar supremo de la misma, ahora se ha desplazado más bien a nuestra representación»².

¿Cómo podemos entender este anuncio? Hay varias interpretaciones del concepto de "muerte del arte» y hay una muy particular que hace Heidegger en el epílogo a $E$ I origen de la obra de arte ${ }^{3}$. Allí dice que lo que viene a indicar la cita, es una pérdida del vínculo entre el Arte y el Ser. Esta desvinculación, que podríamos denominar como una «desontologización del arte», se traduce en una «estetización» del mismo, donde el problema se centra en los medios de representación y de su vivencia estética.

Hegel establecería que después de esta muerte y a pesar de ella, se podrían encontrar dos formas mediante las cuales el arte sobrevivirá a este destino. La primera es a través de la cada vez mayor preocupación por los procedimientos significantes relativos a las técnicas, los procesos y métodos de producción artística. Por otra parte, la progresiva consideración de la obra de arte como canal privilegiado de manifestación de la subjetividad humana, que se va emplazar en el problema de la experiencia del gusto.

¿Dónde se ha ubicado fundamentalmente la arquitectura a partir de esta doble condición? Podría asegurarse que principalmente en el primer ámbito. Es decir, se ha instalado en el campo de los procesos, de los procedimientos, de las lógicas subyacentes a las formas, lo que, en definitiva, la ha vuelto tradicionalmente adicta a una estética del objeto. Recordemos, por ejemplo, la discusión relativa al «buen gusto» en la fundación de la Académie Royale d'Architecture, en $1671^{4}$. ¿Qué ocurre en esa escena?, pues, de hecho, no se trata de una situación cualquiera, porque es la instancia en donde la arquitectura pasa por primera vez a constituirse en disciplina de Estado, es decir, se transforma en un campo formativo y discursivo controlado y al servicio de la Razón de Estado, en este caso, del absolutismo francés. En vísperas de su fundación, se discutía qué es «lo bello» y qué significa el buen o mal gusto en arquitectura. Finalmente y frente al debate sobre el buen gusto, triunfa la teoría de las proporciones, que tiene que ver con una comprensión matematizante de la organización del cuerpo arquitectónico, que subyacería y explicaría su aparición formal. La pregunta es ipor qué triunfa y por qué queda devaluado el problema del gusto? Fundamentalmente porque un discurso vinculado al juicio de gusto respecto de la obra de arte, es una teoría que, ni política ni ideológicamente pertenece a la monarquía n a la aristocracia, sino que es una teoría cuya lógica va a ser principalmente burguesa. Por lo tanto, ¿qué es más acorde para el poder, en este caso, absoluto? Obviamente, una teoría de las proporciones, porque su carácter es normativista y cuya intención principal era la de establecer ciertas condiciones vinculantes en la producción arquitectónica, tomando en cuenta que los intereses que en ese momento tenía la Academia era, entre otros, la creación de un estilo nacional.

Al producirse una suerte de desplazamiento con respecto al gusto, la arquitectura queda más bien capturada en una estética de la obra, que descansa en el eje de la objetualidad. Desde entonces, el discurso arquitectónico se reafirma en su instalación institucional mediante una estética clásica, permitiéndole a futuro evadirse de incorporar de manera decisiva el problema moderno del juicio del gusto.

Posteriormente con «la teoría de la pura visualidad» de Konrad Fiedler, base de la estética del Movimiento Moderno, quedará definitivamente zanjado el asunto de que una reflexión sobre el gusto no es en absoluto necesaria a la hora de comprender la obra arquitectónica, puesto que aquella es más bien una teoría destinada a establecer cuáles son los marcos de la percepción visual ${ }^{5}$.

Hegel, G.W.F. Lecciones de estética. Barcelona: Ediciones Península, 1989; p. 17

Heidegger, M. «El origen de la obra de arte». Arte y poesía. México, Fondo de Cultura Económica, 1958.

Kruft, H-W. Historia de la teoría de la arquitectura 1. Madrid. Alianza Editorial, 1990. / Szambien, W. Simetría gusto, carácter Teoría y terminología de la arquitectura en la época clásica. 1550-1800. Madrid: Ediciones Akal, 1993.

Fiedler, K. De la esencia del arte. Selección de sus escritos realizada por Hans Eckstein. Buenos Aires: Editorial Nueva Visión. Una teoría de la pura visibilidad, por tanto, centrada en rasgos que podríamos identificar como fenomenológicos, no haría suficiente justicia a la subjetividad, constantemente eludida por la arquitectura como vino ocurrir con el problema del gusto. El dominio de la forma pensada a partir de los fenómenos perceptuales, siempre tuvo pretensiones de constituirse en ciencia experimental con rendimientos objetivistas y no discursivos, enjuiciantes o narrativos, como sí acontecería con toda estética de la recepción de impronta kantiana. La pura visualidad vendría, más bien, a reemplazar la teoría de las proporciones que estudia lo que «subyace» al objeto arquitectónico, por una teoría del fenómeno que estudia, esta vez, lo que «construye» perceptualmente al objeto. A pesar de la diferencia, lo que aquí permanece continuo es la gravitación objetual de comprensión, más allá del claro rendimiento subjetivista que la pura visualidad tiene de suyo, en tanto soporte de la conformación espacio-sensorial. Es más, se trata de una teoría no únicamente perceptual sino que, además, se ocupa de privilegiar la visión como sentido primordial de la captura objetual y formal, gesto que, por lo demás, es eminentemente clásico. 
¿Qué pasa con esto?, finalmente toda la arquitectura, desde su impronta clásica hasta el Movimiento Moderno, siempre permanecerá fundamentalmente asociada a una estética de objeto. Ahora bien, ¿qué ocurre posteriormente y cuál sería el escenario en la arquitectura digital? Yo creo que la arquitectura digital es primordialmente continua con esta estética objetualista y, por ende, clásica. Es continua porque arranca de la teoría tradicional de la mímesis, es decir, la posibilidad de asimilar no tanto la forma externa de los objetos, como los procedimientos a través de los cuales se producen aquellas formas. Esto es sustancial al discurso clásico, el ser una comprensión eminentemente procedimental y que es justamente el marco desde donde son pensados básicamente, hoy en día, los recursos digitales.

El discurso digital está ingenuamente aún muy capturado por esta vieja tradición arquitectónica, que tiene que ver con una estética del objeto, en donde lo que interesa es estudiar más bien los procesos subyacentes matematizados -o algoritmizados en este casoque sostienen y constituyen a la forma. Frente a ese tipo de planteamientos arquitectónicos, hay otra vía de comprensión de la arquitectura que está mucho más sintonizada con la vida cotidiana, con la experiencia, con la conflictual condición de la subjetividad actual, que serían de alguna manera todas estas recuperaciones que se han hecho del situacionismo ${ }^{6}$, y cuya vía de comprensión estética es completamente anticlásica y antiobjetual, en definitiva, antirrepresentacional, puesto que el núcleo de su crítica gira en torno a la estética moderna de corte kantiano, estética del gusto que siempre fue eludida o reprimida por la tradición teórica. Por ello que el tenor de la crítica situacionista parecía imposible, en sus inicios, de realizarse desde la institución arquitectónica. La prueba está que todos sus ataques apuntaron al Movimiento Moderno.

Ahora, el divorcio entre parametrización algorítmica y experiencia dibujan, a grandes trazos, más menos el campo contemporáneo de la arquitectura y no hacen sino reeditar la vieja disyunción de la academia, entre teoría de las proporciones y buen gusto, entendiendo que éste último se inscribe de lleno en el problema de la experiencia. Si no se hace una arqueología histórica y estética de las arquitecturas digitales, caeremos

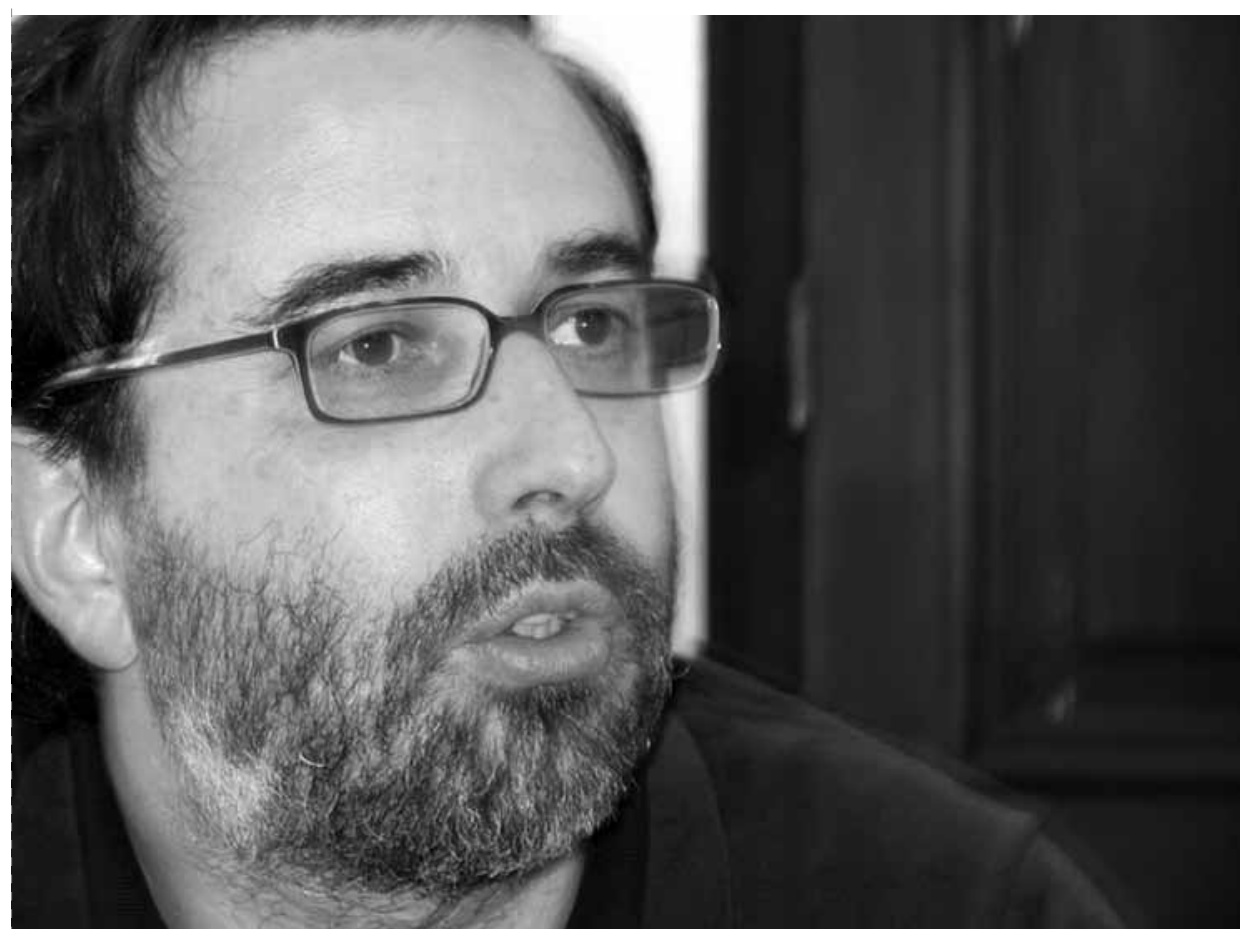

inevitablemente en un debate inmanente centrado en los procedimientos, por tanto clásico y academicista, y sin ninguna capacidad de poder vislumbrar la dimensión social, política e ideológica que tendrían la utilización de los recursos digitales en arquitectura.

-Alberto Montealegre B.: Coincido con el punto de vista hegeliano. Pero no estoy muy de acuerdo, en que la crítica a estos medios debiéramos hacerla exclusivamente desde la perspectiva estética y no darle por lo menos crédito a lo que ellas mismas hacen. En e fondo, la característica que les atribuye su principal interés es la dimensión estética que poseen. Son modelos interesantes en sí mismos, son atractivos, su efecto de seducción es su principal elemento a favor.

El problema está en ¿a quién sirven? En su dimensión técnica la arquitectura siempre se ha caracterizado por una cosa que la diferencia del resto de las artes, y es que siempre ha estado sometida a un encargo, a un encargo contractual. Cuando yo hago algo, lo hago dentro del marco que establece este encargo contractual. Desde Vitruvio que la cosa es así ¿qué es el Tratado de Vitruvio?7 , es toda una reflexión sobre cómo un correcto arquitecto cumple el encargo y cuál es la diferencia entre un arquitecto y el no arquitecto. El arquitecto es el que cumple el encargo y lo cumple bien, a tiempo y en el costo. Esa es la pregunta que uno tendría que hacerle a cualquier sistema nuevo que se relacione con los medios de producción, ¿a quién sirve?, ¿cómo cumple su encargo?, ¿cuál es su encargo? y ¿cómo cumple su encargo? Cuando uno se encuentra frente a esa pregunta no está tomando el tema plástico ni estético, sino que está yendo al origen mismo del problema más pedestre de la arquitectura.

Es justamente ahí donde estos mecanismos están proponiendo una novedad o diciendo incluso en algunos discursos más valientes: "esta es la forma futura de hace arquitectura y la otra la bidimensial está obsoleta, no pueden acompañar al hombre contemporáneo». ¿Cuál es el encargo del hombre contemporáneo? ¿Cómo se insertan estos recursos concretamente en el cumplimiento del contrato? Yo creo que esa

Con respecto al tono de estas recuperaciones por parte de los intereses y condiciones del discurso arquitectónico postmoderno, ver: Solís, J. «El entusiasmo situacionista: arquitectura del espectáculo, espectáculo de la arquitectura». Revista De Arquitectura (Facultad de Arquitectura y Urbanismo, Universidad de Chile) $2005 ; 12$.

De Architectura, tratado sobre arquitectura escrito por Marco Vitrubio (arquitecto romano del siglo I a.C.) considerado el más antiguo que se conserva sobre la disciplina. Según el autor, la arquitectura se define a partir de tres principios básicos: la Belleza (Venustas), la Firmeza (Firmitas) y la Utilidad (Utilitas). 


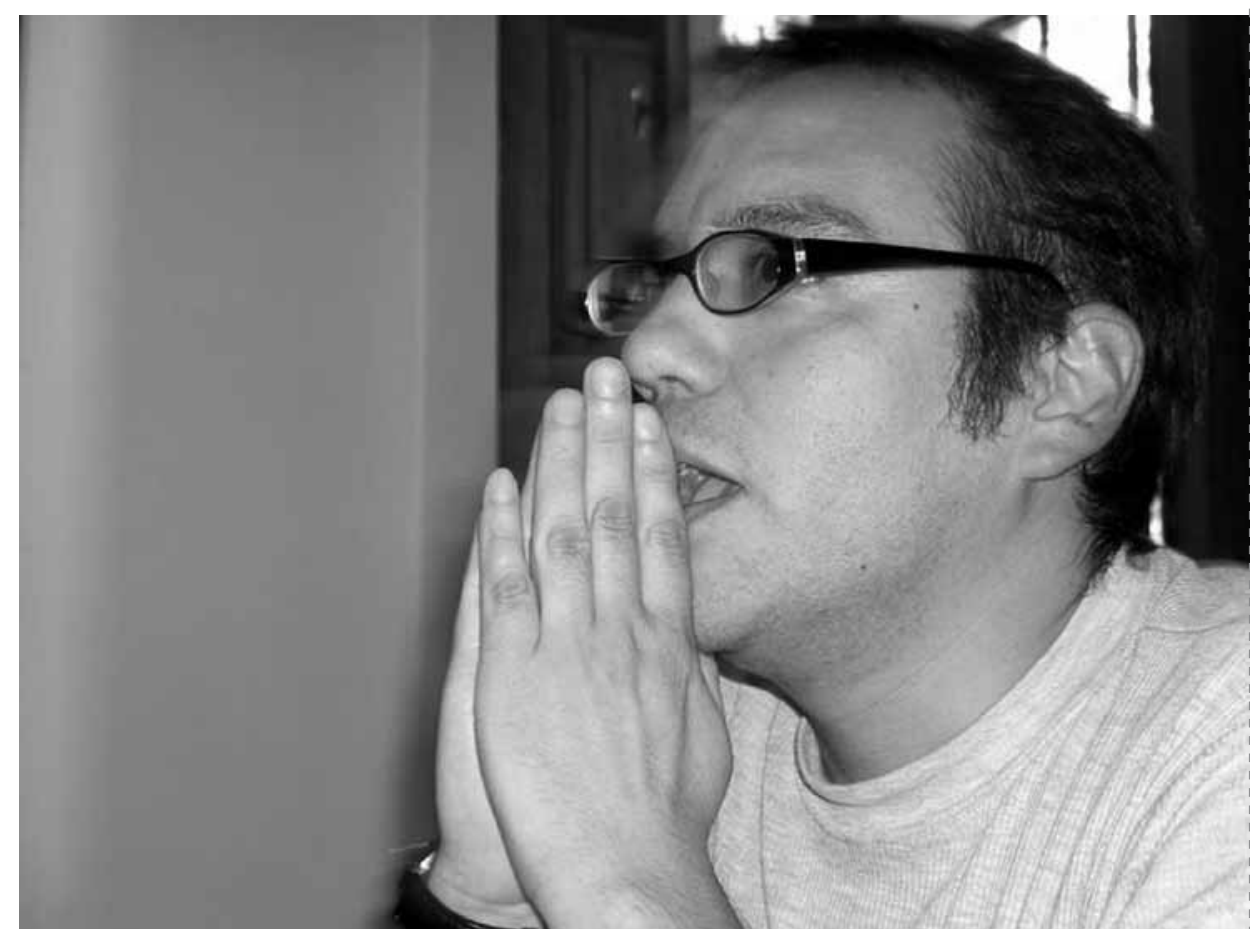

reflexión, por muy pedestre y decepcionante que pueda ser, es fundamental a la hora de juzgar la arquitectura. Ahí está la diferencia; porque creo que el punto de vista estético, tiende a salvar o no hacer la crítica que le corresponde a estos elementos, a estos medios de producción o a estas herramientas. No hace la crítica porque, en realidad, solamente se queda o tiende a quedarse con la visión de la arquitectura como una parte de las artes visuales y no como ese otro aspecto que es anterior y que es el único que nos permite verdaderamente, me parece a mi, hacer un juicio justo sobre la arquitectura.

Es bello, es sensacional el edificio, pero ¿cuándo se hizo?, ¿cómo se hizo?, ¿y en qué momento se hizo? Las condiciones materiales, históricas, temporales, económicas en las cuales se hace una obra, los recursos mecánicos que se emplearon para poder hacerlos son esenciales a la arquitectura. Entonces la crítica que nosotros podemos hacer de la arquitectura, necesariamente tiene que hacerse cargo de esa realidad tecnológica de la arquitectura, de ese fenómeno técnico, y luego podremos apreciar el verdadero mérito que pueda tener una obra o no.

Si nosotros sacamos del problema arquitectónico al problema técnico, que es el problema de las condiciones materiales de su producción, no estamos haciendo una crítica arquitectónica, estamos haciendo una crítica que le sirve a las instalaciones, a la y en ese sentido ahí hay claramente una diferencia entre lo que es la arquitectura y las artes visuales. Cumple primero un encargo contractual y esa es la crítica que tendríamos que hacerle a los medios, a estas herramientas y ahí está la pregunta: ¿A quién sirven?

—José Solís 0.: Creo que efectivamente hay un problema de «sentido», pero el problema del sentido con respecto a los medios digitales, no es por una especie de inadvertencia, de error, o de déficit atencional de quienes hoy día tratan de hacer teoría respecto de esto. Yo creo que tiene que ver con una condición epocal, contexto fundamental para entender por qué el problema del sentido de la reflexión sobre los soportes digitales, no está presente. Cuando hablo de sentido, me refiero a lo que tú acabas de nombrar: contexto social, político, económico y que están vinculados sobre todo al ámbito de la localidad. Lo que hay es una crisis del sentido, pero no una crisis relacionada a la utilización de los recursos digitales, sino que una crisis de sentido con respecto a los fines de la arquitectura como tal, aquello que Lyotard denominaba como pintura, a la escultura, a las artes visuales la muerte de los metarrelatos ${ }^{8}$. Lyotard dice, ¿qué pasa con el ejercicio de la ciencia en la posmodernidad?, ¿cómo se legitima su hacer? La ciencia ya no se pregunta sobre la verdad o falsedad de su campo de investigación o su objeto. Tampoco se pregunta si la investigación que va a realizar es justa o injusta, sino que a lo que tiene que responder es si es eficiente o no. La legitimización que obtiene una acción científica, es de acuerdo a la capacidad que ésta tenga de poder mejorar su actuación o su performance. Eso significa que, derrumbados los fines modernos de la ciencia inspirados por el mito del progreso, se produce una traslación de énfasis que se desplaza hacia los medios o, mejor dicho, la transformación de los medios en los fines de la investigación científica.

Si nosotros tomamos ese mismo fenómeno y lo llevamos a la arquitectura, vamos a encontrar algo similar. Ejemplo de ello es lo que Peter Eisenman en el Fin de lo clásico ${ }^{9}$ establece en relación a la necesidad de que la arquitectura ya no tenga por qué responder a razones externas a su propio discurso, insistiendo en la autonomización del mismo. Declara que la arquitectura no tiene que someterse o legitimarse con respecto a ninguna una alteridad, como sí le ocurría a la ciencia con respecto al mito progresista. Es necesario, entonces, que la arquitectura borre y disuelva el discurso emancipatorio, lo que va a producir exactamente el mismo fenómeno ocurrido con la ciencia postmoderna, es decir, una transición de la legitimidad por los fines hacia los medios arquitectónicos.

Lo que va a ocurrir con ello es que, finalmente, el debate de la teoría de la arquitectura se empieza a reducir a una teoría del diseño y de la proyectación. Hay un énfasis en los procedimientos y los recursos mediante los cuales se produce arquitectura, que viene a dominar la reflexión teórica de los arquitectos. Este es, precisamente, el escenario en el cual ingresan los medios digitales. Y eso se da en un contexto político puesto que, allí donde los fines de la arquitectura se han retirado, se produce un repliegue generalizado de la arquitectura con respecto a la dimensión político-crítica, una despolitización de su discursividad, y en esta situación, pareciera que lo único que queda es que finalmente los recursos digitales sean pensados solamente dentro de la órbita de la representación, del ámbito del modelamiento y los significantes, es decir, en el ámbito de los medios.

\footnotetext{
8 Lyotard, J-F. La condición postmoderna. Madrid: Ediciones Cátedra, 2000.

9 Eisenman, P. «El fin de lo clásico». En: Hereu, P., Montaner, J.M., Oliveras, J. Textos de Arquitectura de la Modernidad. Madrid: Editorial Nerea, 1994.
} 
Podríamos decir que las teorías digitales o sobre lo digital, se inscriben en este campo de autonomía discursiva propia de la arquitectura postmoderna, que tiende a concentrarse más en los medios que en los fines. Que el discurso arquitectónico quede encerrado en los medios va a conducir, a mi juicio, a que finalmente las reflexiones sobre lo digital sean monopolizadas por aquel marco teórico que tiene más sintonía, justamente, con el carácter procedimental de los recursos digitales, que es la teoría de sistemas. Llama la atención que la teorización dominante sobre lo digital, que discursivamente insiste y aplaude a la transdiciplinariedad y la multiplicidad, termine completamente cooptada y protegida principalmente por un solo campo de comprensión, «la teoría de sistemas» que, precisamente, sabe muy bien administrar la diferencia y la complejidad, pero sólo a su modo, según su específico modo, él mismo también «disciplinario». En otras palabras: administra, bajo ella, a la transdiciplina y lo múltiple, pero se erige como marco teórico último, con poco humor para hacer concesiones a otros enfoques que podrían, eventualmente, ocupar su sitio ${ }^{10}$. En términos generales, esta visión dominante de lo digital es primordialmente angloamericana y marcadamente positivista, sin duda le hace justicia a su tradición y la defiende de manera bastante entusiasta.

-Alberto Montealegre B.: Yo creo que hay una especie de mito incluido, y es que, para ponerlo en un término un poco más caricaturesco, el arquitecto solo es incapaz de hacerse cargo de la complejidad del problema del habitar humano, de la solución arquitectónica poniéndola con mayúscula y entre comillas. Requiere de la asistencia computacional. Esa es la gran promesa de los medios digitales, que estas tecnologías permitirán que surja una solución mucho más satisfactoria, por el solo hecho de hacerse cargo de mucha más complejidad de información y además que surja basada en una suerte de adaptabilidad casi ecológica dentro de los espacios ficticios que forman los algoritmos.
Ese es el mito que esta detrás de esta cuestión y que creo tiene sentido en la medida en que nosotros creamos que la información esta ahí y que lo que tenemos que hacer es mejorar la herramienta algorítmica para incorporarla. Pero en realidad la cuestión que no se advierte es ¿Para qué y en qué tiempo soy capaz de sacar una respuesta? ¿Con qué tiempo real soy capaz de esperar a una máquina? La máquina se tiene que detener en un tiempo útil, si la máquina se dedica a iterar infinitamente deja de prestarme utilidad. Cuando esto lo llevamos al mundo del proyecto común y corriente, ni por muy complejo que sea el proyecto, finalmente siempre hay una estructura de gobernabilidad dentro de un proyecto que depende del grado de compromiso que se tenga con respecto al Contrato y el para qué de lo que se está haciendo, el encargo contractual. Siempre hay alguien del otro lado que se da por satisfecho con la solución, finalmente siempre hay un plazo que cumplir siempre hay condiciones reales e históricas de ese problema en particular, no abstractas.

-José Solís 0.: En relación a lo que dice Alberto, vuelvo a repetir que, si no se evalúa el sentido, si no se evalúa el para qué de lo digital, la reflexión va a quedar reducida a una ideología, aquella que tiende a privilegiar y a afirmar como algo intrínsecamente deseable a la globalización de los sistemas de información y comunicación, sin importar los porqué, el para qué y, sobre todo, el para quiénes, lo cual a mi me parece que es, en sí, una toma de posición ideológica -sea ésta consciente o no- que por cierto legítima las razones e intereses de los «hiperconectados». Bauman ${ }^{1}$ nos dice que para sostener la globalización y, por tanto, hacer posible la liquidez del capital internacional y la hiperconexión, es necesario, al mismo tiempo, encerrar en la localidad las consecuencias sociales y políticas desastrosas del transnacionalismo, asumiendo el Estado nacional la función de vigilar que los locales y sus reivindicaciones, no intervengan en la libertad de movimientos de los globales Efectivamente, el discurso teórico respecto a tecnologías digitales tiende a plegarse a esta visión ideológica dominante, que celebra acríticamente la globalización económica y comunicativa y que es, particularmente, adicta a la teoría de sistemas. Creo que es necesario construir un debate que podríamos denominar como «geopolítica de los medios digitales», asociado a discutir el cómo los países periféricos -como el nuestro- requieren traducir los recursos digitales, más allá de una reflexión clásica y procedimental de los mismos, que reproduce los modos de comprensión propios del primer mundo. Nuestra discusión es distinta a la de éste, y creo que muchas veces las problematizaciones que se instalan a nivel local sobre lo digital, provienen de un contexto de intereses sociales, políticos y económicos que no son asimilables a nuestra situación periférica, y debido a ello, vuelven el debate algo extremadamente academicista y autónomo. Ahora, sin duda éste sería una discusión que no podría estar ajena a la academia y a la universidad.

-Alberto Montealegre B.: La pregunta que uno le puede hacer a estos sistemas de modelamiento es "Muéstreme resultados. Dígame para qué se utilizó este dato y cómo eso mejora concretamente una solución de diseño que yo no podría ya haber anticipado solamente con mi lápiz grafito».

Ese es el aterrizaje al mundo en el que vivimos realmente, el cotidiano ¿Cómo entra esto? ¿Cómo incorporarlo? Y por eso pregunto ¿Qué importancia darle en la formación de arquitecto? Porque ya que existe, tenemos que hacernos cargo de él. Pero, ¿necesitamos un taller de diseño por este método, o lo necesitamos como un curso formativo paralelo a otros cursos formativos, equivalente en créditos al curso de Figura Humana? ¿Cómo se introducen estas nuevas metodologías en una Universidad como la Universidad de Chile, que se plantea como una Universidad Nacional, que es una Universidad para el país. ¿Cómo se responde esto? ¿Cómo se inserta? A lo mejor una universidad privada, se puede dar el lujo de dedicarse exclusivamente a esta cuestión. La cuestión está en como aquí, en la Universidad de Chile, nos hacemos cargo de este tema.

—José Solís 0.: Exactamente, creo que es muy importante que esta Universidad se haga cargo precisamente de ese debate. Por ejemplo, ¿qué pasa con las metodologías de investigación social que son de orden cualitativo y que no pueden reducirse a datos algoritmizables? Aparentemente dichas métodos no podrían ingresar en estos nuevos sistemas, a menos que se puedan parametrizar. Estamos frente al debate entre la utilización de recursos digitales y el dominio de endimientos a la eficiencia de la gubernamentalidad -en el sentido foucaultiano del término- sobre todo desde las visiones neoliberales respecto de políticas de Estado. Cibernética, teorías del Caos y de los juegos, todas mercados, sias a la de Sistemas, serían ad hoc en la administración eficaz de la complejidad, ya no sólo de los mercados, sino tambèn de lo polico-social, en tanto se Rodríguez, D., Arnold, M. Sociedad y Teoría de Sistemas. Santiago: Editorial Universitaria, 1992; y Balandier, G. El desorden. La teoría del caos y las ciencias sociales. Barcelona: Editorial Gedisa, 2003.

11 Bauman, Z. La globalización. Consecuencias humanas. Buenos Aires: Editorial Fondo de Cultura Económica, 1999. 


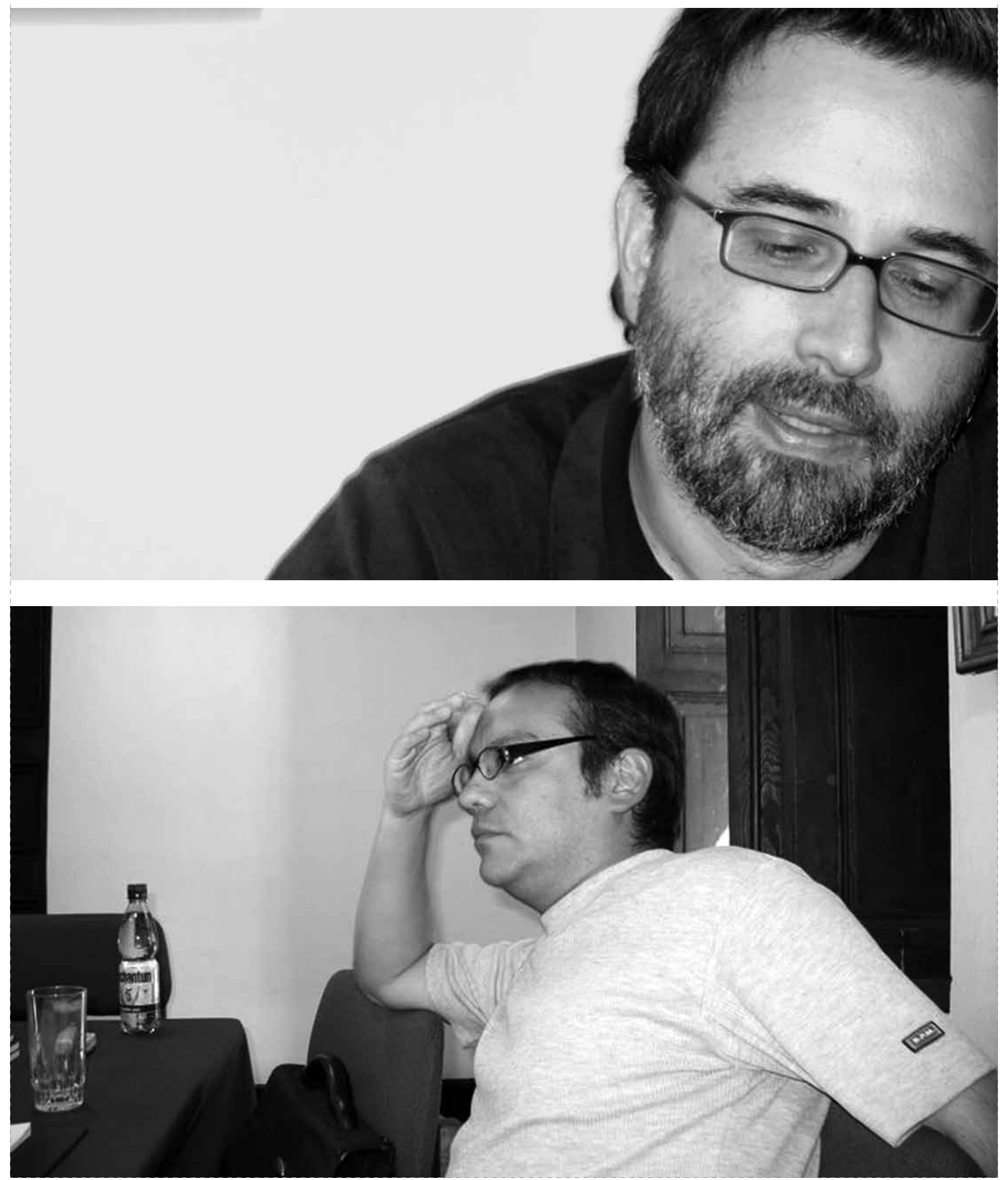


la simbolización, el dominio de la experiencia, el campo del sentido, precisamente, el de la vida cotidiana. ¿Qué importancia tendría esto para el planeamiento urbano, para la acción del Estado, cuales sería sus proyecciones políticas o más aún, biopolíticas? ${ }^{12}$. O nos abandonamos a los debates inmanentes centrados en los recursos de representación y modelamiento propios del clasicismo primer mundista, o nos abrimos a lo que yo creo es central en esta discusión, que es la diferencia o el divorcio entre, por un lado, la esfera del sentido que siempre está instalado en la localidad y lo cotidiano y, por otro, esta escena globalizante, hiperconectada de las arquitecturas paramétricas.

Ese divorcio hay que pensarlo, yo no estoy diciendo que no puedan haber posibilidades de sincretismos, pero incluso para que ellas efectivamente ocurran, tenemos que hacernos cargo primero de este hiato existente hoy en día, que tiene que ser pensado en la Escuela de Arquitectura de la Universidad de Chile, si no en qué otro lugar, siempre y cuando partamos del supuesto de que éste es un espacio crítico a las posiciones dominantes, de lo contario, no tiene ningún sentido esperar tal debate, al menos aquí.
-Alberto Montealegre B.: Estoy de acuerdo, es esencial que una Escuela de Arquitectura se piense a si misma y se plantee cuales son las competencias que pretende formar. Si no en realidad pasa que se cae de lleno en el terreno estético y al caer de lleno en el terreno estético estamos perdidos, porque la cantidad de discursos que pueden aparecer seductores sobre esto es enorme. Es decir, ahí donde el no controlar el código fuente del software nos hace sometibles. Siempre nuestro quehacer va a estar controlado por la vanguardia, siempre nuestro quehacer va a caer en la esfera de los productos de consumo. Por lo tanto, todo lo que hagamos va a tener ese sello de lo caducable y nunca vamos a tener elementos de juicio que le pertenezcan al problema, al objetivo final.

Es necesario pensar ¿Para qué es esta Escuela de Arquitectura? ¿Cuáles son las competencias de los profesionales formados aquí, para saber en qué medida estas temáticas entran y cómo podemos aprovecharlas? Es necesario para la Universidad de Chile incluir este tipo de reflexiones, que tiene que ver con la competencia para poder verdaderamente sacarle provecho a estos nuevos recursos.
Hacer algo útil con ellos. Si no somos capaces de encontrar esa pauta...., esto es ruido, es sólo seducción, es sólo estética.

-José Solís 0.: Hay que superar esta pasión adolescente por el plano únicamente significante, experimental y procedimental de los medios digitales, porque no nos deja ver lo importante que ellos tienen. De ningún modo hay que negar la presencia de estos nuevos medios, hay que hacerse cargo desde nuestra localidad, con nuestros problemas $y$, en ese sentido, hay que asumir y comprender nuestra presencia periférica frente a los campos de producción tanto simbólica como comercial de dichos recursos. Transitar desde esta fascinación por los soportes digitales en términos de su capacidad de modelamiento, esta suerte de énfasis en los medios más que en los fines y concentrarse en el problema del sentido, es la discusión de fondo, una discusión que yo diría es eminentemente socio y geopolítica.

12 El concepto de biopolítica ha sido acuñado por Foucault, para referirse a la particular forma de gubernamentalidad en donde la inscripción de los procesos vitales de los ciudadanos, devenidos en población, pasan a convertirse en uno de los aspectos centrales de la gestión del Estado Moderno. Con respecto a las lógicas de agenciamiento poblacional, ver sobre todo: Foucault, M. Seguridad, territorio, población. Buenos Aires: Editorial Fondo de Cultura Económica, 2006; y sobre las condiciones de su surgimiento: Nacimiento de la biopolítica. Buenos Aires: Editorial Fondo de Cultura Económica, 2007; ambos textos correspondientes a los cursos dictados por el autor en el Collage de France entre 1977 y 1979 . Si comprendemos sobre todo que la bíopolítica no se reduce exclusivamente al dimensionamiento biológico del individuo y de la población, sino a la constitución de una competitividad vital, habría que ligarla a la noción de «vida cotidiana», con todas las consecuencias que ello significa tanto para el diseño como para la arquitectura y el urbanismo. 